\title{
The Role of Cytokins in Some Viral Infectious Diseases
}

\author{
Shimon Shatzmiller*, Galina Zats, Inbal Lapidot and Rami Krieger \\ Department of Biological Chemistry, Ariel University, Arieel, Israel \\ *Corresponding Author: Shimon Shatzmiller, Department of Biological Chemistry, \\ Ariel University, Arieel, Israel.
}

Received: July 25, 2020

Published: September 30, 2020

(C) All rights are reserved by Shimon

Shatzmiller., et al.
Cytokines are small glycoproteins produced by several cell types, mainly leukocytes, which regulate immunity, inflammation and hematopoiesis. They control several physiological and pathological roles, including innate immunity, acquired protection, and an abundance of inflammatory responses. The research of cytokines starts in the 1950s. However, the exact identification of the structure and its function took many years to explore. The original findings were those of IL-I, IFN, and neurodevelopment factors (NGFs); However, these cytokines were purified and transferred in their name years later. Research of the exact physiological, pathological and pharmacological effects of some cytokines is still in progress. For example: Neurotrophins are a family of proteins that induce the survival, development, and function of neurons [NGF, BDNF, neurotropin-3 (NT-3) and NT4/5] are secreted proteins that have a strong effect on the neural nerve Survival, differentiation, and synaptic function. Adult neurotrophins bind better to Trans-kinanic (Trk) and p75 NTR, whereas proneurotropins, which contain a proteolytic N-terminal region Removed in "mature" forms, communicates with p75 NTR and through it Their N-terminal domains, with sorting receptor sorting. The p75 NTR interacts with Trks and regulates the Trk signals But it is also independently integrated into various probiotic and prooptotic signaling systems.

Several lines of evidence support the hypothesis that LM11A compounds act primarily through direct interactions with p75NTR: (1) active compounds fail to elicit prose activity and AKT signaling in hippocampal neurons from p75NTR mice -/ -; (2) active compounds appear to interact with p75NTR, as demonstrated by their ability to bypass NGF from p75NTR-Fc but not from TrkAFc; And (3) Ab 9651, an antibody directed to the neurotropin- binding domain of p75NTR, inhibits the effects of complex development and is suitably delivered by active LM11A compounds.

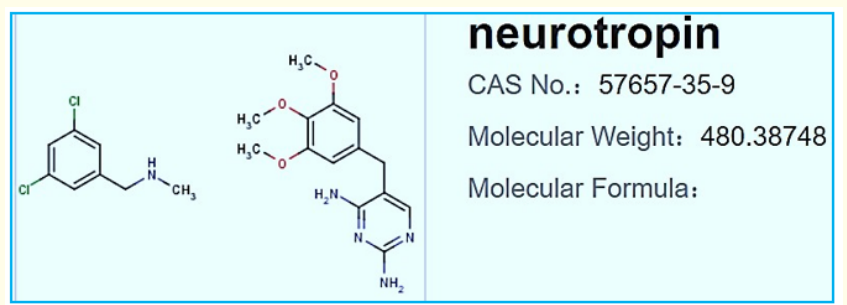

Figure 1

Further support for this claim is given by the findings that active compounds result in rapid recruitment of IRAK to p75NTR, that they do not activate the Trks, and that at maximal doses, NGF and active compounds have no additive effects. Thus, p75NTR appears to be necessary for LM11A compound activity; However, it is possible that the compounds also work to some extent through low-level or alternative modes for trk activation, or through other mechanisms that interact with p75NTR. Finally, it was interesting to note that although they are ostensibly related solely to the feature of a pharmacopoeia containing a mimetic binding of the NGF 1 p75NTR loop, the various LM11A chemical compounds have similar neurotrophic activities and intensities. This observation is also consistent with the possibility that these compounds act via p75NTR (Figure 2).

To extend the evaluation of the compounds from chick DRGs to mammalian CNS neurons, further biologic analysis of the compounds was performed using embryonic mouse hippocampal neurons. Under conditions of low density and lack of glial support, the survival of these neurons is dependent, in part, on the addition of 
neurotrophins. Screening of the 23 previously tested compounds in these cultures demonstrated activity of three of the compounds (LM11A-7, -24, and -31) previously identified as having activity in DRG cultures and identified a fourth active compound, LM11A-28 (structures in figure 2).

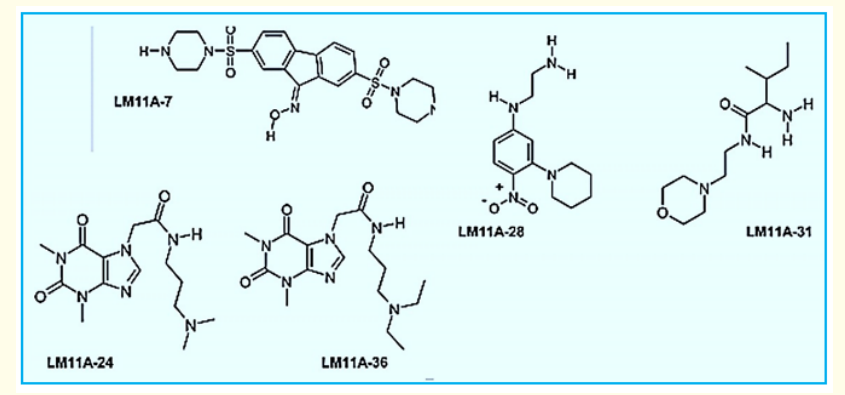

Figure 2

The clinical appearances of COVID-19 vary from: asymptomatic forms or symptomatic inflammation to clinical conditions characterized by respiratory failure requiring mechanical ventilation and intensive care support, to multisystem and systemic manifestations in terms of sepsis, absorption shock, and multiple organ dysfunction (MODS). In one of the first reports of the disease, Huang et al. Illustrated that patients (No. 41) suffered from fever, malaise, dry cough and shortness of breath. Computed tomography scans of the chest showed pneumonia with abnormal findings in all cases. About a third of these $(13,32 \%)$ required intensive care, and there were $6(15 \%)$ fatal cases.

\section{Foreword}

Influenza $\mathrm{A}$ is one of the most important causes of respiratory diseases. It replicates in epithelial cells and leukocytes resulting in the production of immune mediators - cytokines, substances with different biological effects. Cytokines, as part of innate immunity, prefer the development of antiviral and TH1 immune responses. Cytokines also affect the adaptive immune response and disease expression. In the organism, the virus is caused by the production of a chemotactic [regulated with activation, cytokine expressed in normal and secreted T cells (RANTES), chemo-attraction proteins monocytes (MCP) MCP-1, MCP-3, inflammatory protein macrophage 1a (MIP-1a), protein 10 Caused by interferon $\gamma$ (IP-10), and interleukin 8 (IL-8)], inflammatory factor [IL-1 $\beta$, IL-6, IL-18, and tumor necrosis factor $\alpha$ (TNF- $\alpha$ )] and antiviral cytokines [ interferon (IFN) $\alpha / \beta$ ]. Although knowledge about the mechanisms un- derlying host and tissue specificity has progressed significantly, we still know relatively little about the function of the various cytokines released from various cells following influenza infection.

Recognition that inflammation may represent a common mechanism of disease has been expanded to include neuropsychiatric disorders. Including major depression. Patients with palpable depression have been shown to show an increase in inflammatory biological markers in peripheral blood, including inflammatory cytokines, which have been shown to be accessible to the brain and interact with almost any pathophysiological. A field known to be involved in depression, including neurotransmitter, neuroendocrine function and neuroplasticity. Indeed, the assessment that activation of inflammatory pathways in the brain contributes to the encounter of decreased neurotropic support. And altered release/ reabsorption of glutamate, as well as oxidative stress, leading to excitotoxicity and loss of exile elements, consistent with Neuropathological findings characterizing depressive disorders. Even more represents the link between inflammation and depression Data showing that psychosocial stress, a known fluid of mood disorders, is capable of stimulating inflammatory signaling. Molecules, including the Capa B nuclear factor, in part, by activating flow pathways of the sympathetic nervous system. interesting, Patients with depression with an increase in inflammatory biological markers were found to be more likely to show resistance to treatment and in 2002. In several studies, antidepressant medication has linked to a decrease in inflammatory responses. Finally, preliminary data are applied. With inflammatory disorders, as well as medically healthy depressed patients, suggest that inhibitors of pro- inflammatory cytokines or their Signaling pathways may improve the depressive mood and increase the response of treatment to conventional antidepressants. The translation implications of these findings include the unique opportunity to identify relevant patient populations, implement vaccine-targeted. Various therapies and monitoring of therapeutic efficacy at the level of the immune system in addition to behavior is in progress.

\section{Cytokines in COVID 19}

COVID-19 is a $\beta$-coronavirus virus caused by infection with SARS-CoV-2 and is closely related to SARS-CoV. This is the third Coronavirus disease that affects humans, following severe acute respiratory syndrome (SARS) and Respiratory Syndrome in the Middle East (MERS). 
The clinical symptoms of COVID-19 appear after the initial incubation period of about 5-6 days. They include frequent fever, cough and fatigue, with the possible onset of sputum production, headache, hemoptysis, diarrhea, disorder, and/or lymphopenia, among others. Computed tomography images of patients with severe COVID-19 complications reveal the presence of pneumonia albeit with abnormal features, including RNAemia, acute respiratory distress syndrome, acute heart damage, and evidence of opacity of the ground glass lung. In some patients, opacity of the ground glass was found in sub- peripheral areas of both lungs, which may lead to systemic and local immune responses, which exacerbates the inflammation. In some cases, inhaled and interferon treatment not only did not have positive effects but also exacerbated the clinical symptoms, with progression to pulmonary opacity. Patients in advanced age and suffering from basic conditions (e.g., hypertension, chronic obstructive pulmonary disease, diabetes and/or cardiovascular disease, etc.) are at higher risk for rapid progression to acute respiratory distress syndrome, absorption shock, metabolic acidosis, coagulation function, disorders Heart rate, kidney damage, heart failure, liver function and/or secondary infection, which usually leads to death.

COVID-19, the disease caused by coronavirus SARS-Cov-2, has infected millions people in most countries, of whom at least 500000 have died. The economic and social impact of the pandemic is staggering, but despite a daily flood of news on the disease, few laypeople know that paradoxically, COVID-19 mostly kills through an overreaction of the immune system, whose function is precisely to fight infections.

"Because treatment for now relies on aggressive treatment of symptoms, preventative protection against secondary infections, such as bacteria and fungi, is particularly important to support organ function, especially in the heart, kidneys, and liver, intestines and brain. This may be achieved by way of the gut-brain axis to try and avoid further deterioration of their condition".

Based on the idea that inflammation caused by microbes that cross the BBB initiating a cascade of events that leads to neuronal death, Harvard scientists try to cure both COVID 19 and Alzheimer's disease by use one drug. A new treatment for Alzheimer's disease now in advanced Phase 3 clinical trials may also be effective in treating pneumonia caused by Covid-19. The research treatment, ALZT-OP1, suppresses the production of the immune system cytokines - proteins that cause inflammation in a natural response to infection. The "cytokine storm" caused by a severe immune response plays a role in neuro-inflammatory diseases such as Alzheimer's and infectious diseases such as Covid 19 and influenza.

"When there is too much of a health virus, our immune system begins to respond by activating cytokines to bombard the virus", explains Harvard-born associate professor and Israeli medical studies professor David Elmaleh, scientific founder and CEO of AZ Therapies.

\section{Assets from publication with us}

- Prompt Acknowledgement after receiving the article

- Thorough Double blinded peer review

- Rapid Publication

- Issue of Publication Certificate

- High visibility of your Published work

Website: www.actascientific.com/

Submit Article: www.actascientific.com/submission.php

Email us: editor@actascientific.com

Contact us: +919182824667 